\title{
Caecal duplication, a case report
}

\author{
Michele Corroppolo, Elisa Pani, Maria Teresa Bortolami, Hamid Reza Sadri, Fabio Beretta, Clara Revetria, \\ Giosuè Mazzero, Enrico Ciardini
}

U.O.C. Chirurgia Pediatrica, Ospedale Santa Chiara, Trento, Italy

\begin{abstract}
The caecum is one of the rarest sites of intestinal duplication cysts. The most common symptomatology includes vomiting, abdominal pain, abdominal distention, palpable mass and rectal bleeding. Most of the duplications are diagnosed within the first two years of life, including prenatal diagnosis. Only few cases of caecal duplication have been reported in the literature up to the present day. We are going to present a case of a five-years old girl with caecum duplication who reached our ward due to abdominal distension with no other symptoms.
\end{abstract}

\section{Introduction}

The pathophysiology of EDCs remained unclear. ${ }^{1,2}$ It is a challenge to the medical practitioner when dealing with an infant with duplication cyst because of the intermittent nature of the disease.

Correspondence: Michele Corroppolo, U.O.C. Chirurgia Pediatrica, Ospedale Santa Chiara, Trento, Italy.

E-mail: michele.corroppolo@apss.tn.it

Key words: Intestinal duplications; caecal duplication.

Conflict of interest: The authors have no conflict of interest to declare.

Availability of data and materials: All data underlying the findings are fully available.

Ethics approval and consent to participate: No ethical committee approval was required for this case report by the Department, because this article does not contain any studies with human participants or animals. Informed consent was obtained from the patient included in this study.

Consent for publication: The patient's guardians gave their written consent to use the data for the publication of this case report and any accompanying images.

Received for publication: 9 November 2020.

Revision received: 5 September 2021.

Accepted for publication: 22 September 2021.

This work is licensed under a Creative Commons Attribution

NonCommercial 4.0 License (CC BY-NC 4.0).

CCopyright: the Author(s), 2021

Licensee PAGEPress, Italy

La Pediatria Medica e Chirurgica 2021; 43:246

doi:10.4081/pmc.2021.246
Gastrointestinal duplications are congenital malformations that can involve any part of the Gastrointestinal (GI) tract from the mouth to the anus. The incidence of enteric duplication cysts is reported to be 1 in every 4,500 live births 1,2 and it is about $1 \%$ of GI tract anomalies. Caecal duplication is very rare: only few cases have been reported in literature. 3,4 The most frequent symptoms are abdominal pain, abdominal distension, palpable mass and rectal bleeding. Most of the duplications are diagnosed prenatally or within the first two years of life. We are going to present the case of a five-years old girl with caecum duplication who reached our ward due to a significant abdominal distension with no previous diagnosis of intestinal duplication.

\section{Case report}

A five-years old girl reached our Emergency Room due to a significant abdominal distension present for about 2 months with absence of any other symptoms such as rectal bleeding, vomiting or abdominal pain or constipation. On physical examination, the abdomen was globular, soft and non-tender, painless on superficial and deep palpation and hypochondriac organs were within limits as far as assessable. Peristalsis was present and valid, and a significant tympanism at the percussion in epi-mesogastric regions was present. Blood tests were all normal. The general conditions of our patient were good apart from the abdominal distension and that is why an intestinal occlusion was improbable as clinical diagnosis. Thus, an abdominal X-ray was not performed but only an ultrasonography. The abdominal ultrasonogram showed a cyst in the mesogastric region with greater transverse and craniocaudal dimensions being respectively $17 \mathrm{~cm}$ and $9 \mathrm{~cm}$ long with the presence of corpuscular contents and sediments in the inferior part (Figure 1). An abdominal MRI was performed which showed the presence of a mass with a liquid content in the central abdominal quadrant, predominantly occupying the mesograstrium, left and right pelvic regions. Cyst dimensions were approximately $16 \mathrm{~cm}$ major latero-lateral axis, $6.5 \mathrm{~cm}$ antero-posterior and approximately $8.5 \mathrm{~cm}$ craniocaudally starting from the mesenteric root of the right pelvis (Figure 2). We conducted a median laparotomy, which confirmed the presence of an enormous and thick-walled cystic formation. We proceeded with the insertion of an $8 \mathrm{Ch}$ catheter inside the neoformation, securing its insertion point with a pursestring suture to avoid any spillage. Endo-cyst fluid sample was sent for extemporaneous cytological examination, which was negative for presence of malignant cells. Thus, the cystic content was drained $(780 \mathrm{~mL})$, allowing recognition that the cyst arose from the caecum (Figure 3). En-bloc resection of the neoformation with the last $3 \mathrm{~cm}$ of ileum, caecum, appendix and of approximately 5 $\mathrm{cm}$ of the ascending colon was performed, followed by an ileocolic termino-terminal anastomosis and closure of the abdominal 
wall. Sampling lymph node of the ileocecal corner showed reactive hyperplasia. The histological examination confirmed the operative suspicion of intestinal duplication.

\section{Discussion}

Gastrointestinal duplications are congenital malformations that can occur anywhere along the alimentary tract. Ladd in 19375 introduced the term "duplication of the alimentary tract" and Gross in $1953^{6}$ defined their clinical and pathological features. According to Ladd and Gross, the intestinal duplications have an intimate anatomical association with some portion of the gastrointestinal tract, being covered with epithelial lining representing some portion of the gastrointestinal tract, a presence of a welldeveloped coat of smooth muscle and sharing blood supply with

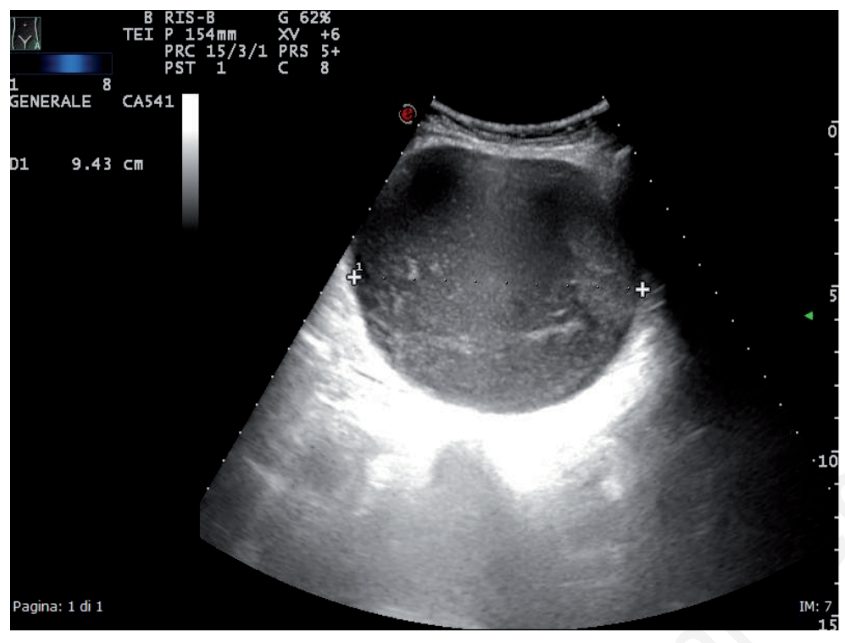

Figure 1. Abdominal ultrasound showing the cyst with approximate dimensions of $17 \mathrm{~cm} \times 9 \mathrm{~cm}$.

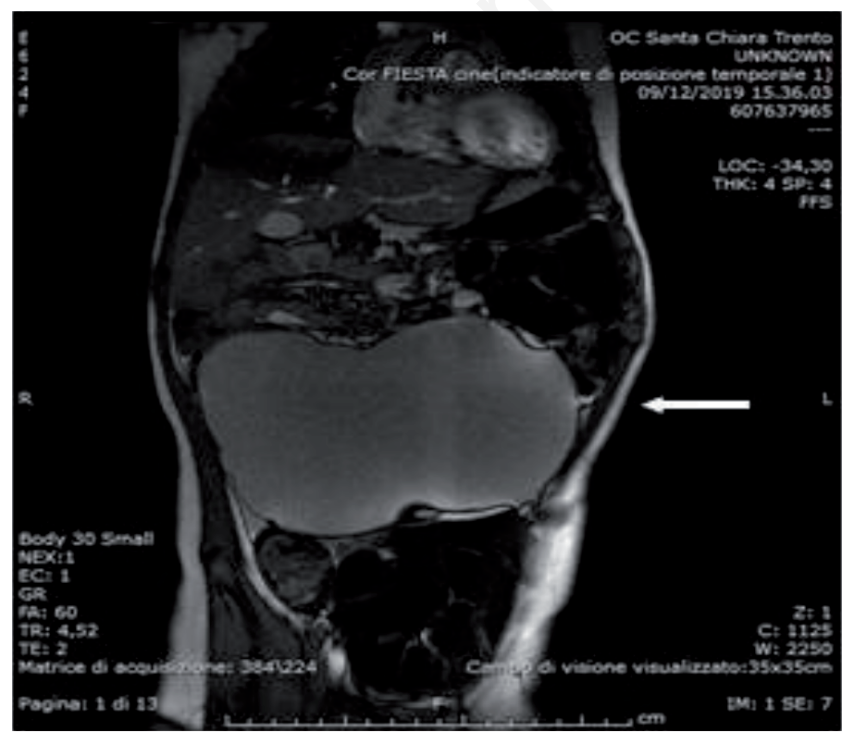

Figure 2. MRI of abdomen, the arrow shows the cyst with approximate dimensions of $16 \times 6.5 \times 8.5$. the native intestine. Depending on their morphology, we can distinguish between tubular duplications and cystic duplications.

Enteric duplication cysts are a group of heterogeneous clinical entity with varied clinical presentations due to the size, location, type and mucosal pattern of the cysts. They are thought to occur between the 4th and 8 th week of embryonic development. ${ }^{2}$ The aetiology of enteric duplications remained unclear. Duplication of the ileum is the most common (33\%), followed by oesophagus $(20 \%)$, colon (13\%), jejunum (10\%), stomach (7\%) and duodenum $(5 \%) .1,2$

Duplication of the caecum is very rare $(3,4)$. Oudshoorn 7 has reviewed 362 cases of duplication cysts reported in the literature and found only 16 cases of caecal duplications. Presenting symptoms in these cases are as follows: abdominal mass, intussusceptions, gastrointestinal haemorrhage or abdominal pain mimicking appendicitis. It can also cause chronic or acute intestinal obstruction.

The exact aetiology of the enteric duplications is unknown. Enteric duplication cysts can occur at any age, in $80 \%$ of cases within the first two years of life. Furthermore, it may also occur in association with a clinical picture of intestinal obstruction 8,9 due to invagination, ${ }^{10}$ volvulus or compression of the cyst itself.

We did not perform a formal right hemicolectomy because in these cases it is not indicated or necessary; in fact, surgical procedures of small cystic or short tubular duplication involve segmental resection, along with adjacent intestine, with a primary end-toend anastomosis.

The prognosis of these type of malformations is very good.

In our case, the peculiarity was the presentation with abdominal distension and no other significant clinical manifestations. Ultrasonography was carried out in an attempt to obtain a more accurate pre-operative diagnosis, which was actually achieved at the operation and confirmed by subsequent post-operative histological examination.

Whenever there is a cystic lesion in right abdominal quadrants on ultrasonography in neonates and children, despite the absence of a palpable abdominal mass, enteric duplication cyst is to be considered in the differential diagnosis, whether it is the most common ileal variant, or like in our case, the rarest caecal duplication cyst would be obvious only during the surgery.

The most common imaging modalities to diagnose duplication cysts are ultrasonography and CT abdomen, but identifying the exact location of cyst is still difficult.

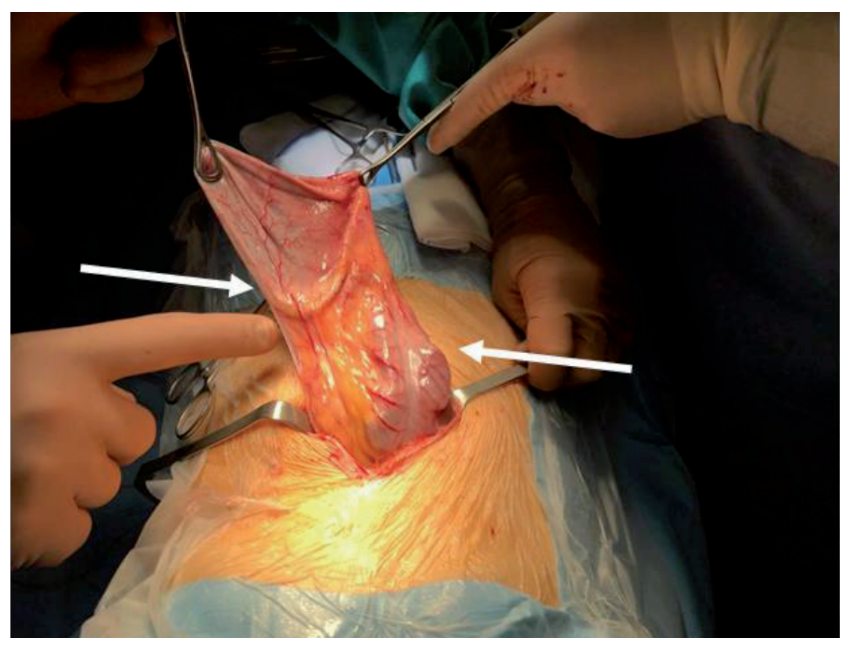

Figure 3. Intraoperative finding, cyst walls (left white arrow) after evacuation of $780 \mathrm{cc}$ of turbid liquid, which origins from the caecum (right white arrow). 
Although most of intestinal duplications present as intestinal obstruction, our case showed only painless abdominal distension.

\section{Conclusions}

Our case was really challenging due to atypical history and clinical presentation. We recommend taking caecal cyst in differential diagnosis of painless abdominal distension in paediatric patients, although it is rare.

\section{References}

1. Cinta SN, Roberto LS, Elena CP, et al. Enteric duplication cysts in children: varied presentations, varied imaging findings. Insights Imaging 2018;9:1097-106.

2. Roy L, Douglas GA. Duplication cysts: diagnosis, management and the role of endoscopic ultrasound. Endosc Ultrasound 2014;3:152-60.
3. Mehl SC, Anbarasu C, Sun R, Naik-Mathuria B. Cecal duplication cyst: a rare cause of pediatric bowel obstruction. Am Surg 2020:3134820951425.

4. Pati A, Mohanty HK, Subudhi PC, et al. Duplication cyst of the cecum: A case report. Indian J Surg 2010;72:271-2.

5. Ladd WE. Duplication of the alimentary tract. South Med J 1937;30:363-71.

6. Gross RE. The surgery of infancy and childhood. Philadelphia: Saunders; 1953. pp. 221-45.

7. Oudshoorn JH, Heij HA. Intestinal obstruction caused by duplication of the caecum. Eur J Pediatr 1996;155:338-40.

8. Keum SW, Hwang MW, Na JI, et al. Intestinal obstruction caused by a duplication cyst of the caecum in a neonate. Korean J Pediatr 2009;52:261-4.

9. Dombale V, Patil BV, Kadam SA, Kerudi BH. Enteric duplication cyst of caecum presenting with intestinal obstruction- a case report. J Krishna Inst Med Sci Univ 2012;1:147-9.

10. Verma S, Bawa M, Rao KL, Sodhi KS. Caecal duplication cyst mimicking intussusception. BMJ Case Rep 2013;2013: bcr2012008056. 